\title{
Article
}

\section{Electrochemical Evaluation of Nitroxyl Radical Catalysts and Electrochemical Detection of Cyclosporin A}

\author{
Sachiko Komatsu 1, Yusuke Sasano ${ }^{2}$, Kyoko Sugiyama 1, Kazuhiro Watanabe 1, \\ Masayuki Kumano 2, Kentaro Yoshida ${ }^{3}$, Tetsuya Ono ${ }^{3}$, Yoshiharu Iwabuchi ${ }^{2}$, \\ Tsutomu Fujimura ${ }^{1}$, Katsuhiko Sato ${ }^{1,4}{ }^{,}$, and Yoshitomo Kashiwagi ${ }^{3, *}$ \\ 1 Faculty of Pharmaceutical Science, Tohoku Medical and Pharmaceutical University, 4-4-1 Komatsushima, \\ Aoba, Sendai, Miyagi 981-8558, Japan; sachicom@tohoku-mpu.ac.jp (S.K.), kyoko.sugiyama@tohoku- \\ mpu.ac.jp (K.S.), k-nabe@tohoku-mpu.ac.jp (K.W.), tfujitsu@tohoku-mpu.ac.jp (T.F.), satok@tohoku- \\ mpu.ac.jp (K.S.) \\ 2 Graduate School of Pharmaceutical Sciences, Tohoku University, 6-3 Aoba, Aramaki, Aoba-ku, Sendai 980- \\ 8578, Japan; ysasano@tohoku.ac.jp (Y.S.), masayuki.kumano.p2@dc.tohoku.ac.jp (M. K.), \\ iwabuchi@mail.pharm.tohoku.ac.jp (Y.I.) \\ 3 School of Pharmaceutical Sciences, Ohu University, 31-1 Misumido, Tomita-machi, Koriyama, Fukushima \\ 963-8611, Japan; k-yoshida@pha.ohu-u.ac.jp (K.Y.), t-ono@pha.ohu-u.ac.jp (T.O.), y-kashiwagi@pha.ohu- \\ u.ac.jp (Y.K.) \\ 4 Department of Creative Engineering, National Institute of Technology, Tsuruoka College, 104 Sawada, \\ Inooka, Tsuruoka, Yamagata 997-8511, Japan; satok@tohoku-mpu.ac.jp (K.S.) \\ * Correspondence: y-kashiwagi@pha.ohu-u.ac.jp; Tel.: +81-24-932-9146 (Y.K.)
}

\begin{abstract}
Nitroxyl radical catalysts oxidize alcohols under an applied electric potential. It is possible to quantify the alcohol concentration from the resulting oxidation current. In this work, we evaluated the catalytic activity of nitroxyl radicals (or their corresponding hydroxylamines), including 2,2,6,6-tetramethylpiperidine-1-oxyl (TEMPO) as representative nitroxyl radicals, acetoamido-TEMPO, which shows higher oxidation potential than TEMPO owing to the acetoamido group, AZADO, Nor-AZADO, and NNO as less-hindered bicyclic nitroxyl radicals, and NHPI as an $N, N$-diacyl-type hydroxylamine, in acetonitrile solution. TEMPO, AZADO and NNO were also evaluated for their ability to oxidize alcohols in organic solvents, and their reactivity was compared with the electrochemical response. The most active NNO was used for electrochemical detection of cyclosporin A, a drug with a hydroxyl group.
\end{abstract}

Keywords: 1; nitroxyl radical 2; TEMPO 3; cyclosporine A 4; sensor

\section{Introduction}

Cyclosporin A (CSA), a lipophilic, cyclic peptide containing 11 amino acids [1], has been widely used to suppress rejection in organ transplantation. CSA binds to the T-cell receptor protein cyclophilin, and this complex acts as a calcineurin inhibitor that suppresses cytokine production. CSA is also applied therapeutically to treat autoimmune diseases such as Behcet's disease, aplastic anemia, and nephrotic syndrome. Since CSA has a narrow effective treatment range, determination of the blood CSA levels is required in order to avoid the risk of rejection or organ toxicity. Therefore, therapeutic drug monitoring (TDM) is typically performed during immunosuppressive therapy with CSA. 
Many TDM methods for CSA are carried out by immunoassay or liquid chromatographytandem mass spectrometry (LC-MS/MS), each of which has advantages and disadvantages. Major commercially available kits for determination of CSA levels in whole blood have been developed based on immunoassays, such as the enzyme multiplied immunoassay technique (EMIT), affinity column mediated immunoassay (ACMIA) [2], chemiluminescence immunoassay (CLIA) [3], and electrochemiluminescence immunoassay (ECLIA) [4,5]. Although these immunoassays are simple and reliable, it should be noted that the blood drug levels obtained depend on the antibody and detection principle for each method. In addition, cross-reactions often induce blood level overestimation as compared with LC-MS/MS. The LC-MS/MS method has high sensitivity and specificity, and is thus valuable for simultaneous measurement of immunosuppressants and their metabolites [6-8]. However, LC-MS/MS requires mastering of instrument operating protocols and has a high initial cost.

Electrochemical analysis has been used in various fields due to its high temporal and spatial resolution and simple measurement procedure. Even for compounds that do not show an electrode response, such as glucose and glutamate, a biosensor with high selectivity can be constructed by immobilizing the corresponding enzyme on the electrode surface. Determination of the substrate concentration from the current can be performed by oxidation or reduction of $\mathrm{H}_{2} \mathrm{O}_{2}$ or NADH generated by an enzymatic reaction on the electrode $[9,10]$. However, enzymes, which are biological macromolecules, are expensive and have problems with long-term stability and heterogeneity in production lots. In addition, it is difficult to construct biosensors for molecules such as CSA, which does not undergo enzymatic reactions. Therefore, we have investigated the use of organocatalysts such as 2,2,6,6-tetramethylpiperidine $N$-oxyl (TEMPO) instead of enzymes to determine the substrate concentration based on an electrical signal [11]. TEMPO is a stable free radical. In organic synthesis, it is used in conjunction with suitable re-oxidizing agents for the oxidation of alcohols [12-14]. In electrochemistry, the oxidation of alcohols is catalyzed by the application of an electric potential under basic aqueous conditions or in organic solvents $[15,16]$. Since the oxidation current obtained is proportional to the alcohol concentration in the solution, it is possible to quantify alcohols and compounds with hydroxy groups in the molecules (Figure 1) [17]. In this study, we initially evaluated the electrochemical oxidation capacity of alcohols in acetonitrile for various nitroxyl compounds. The ability to oxidize alcohols in organic solvents using an oxidizing agent instead of applying a potential was evaluated and compared with the electrochemical response. Electrochemical detection of CSA, a drug with a hydroxyl group in the molecule, was carried out using the most active NNO.

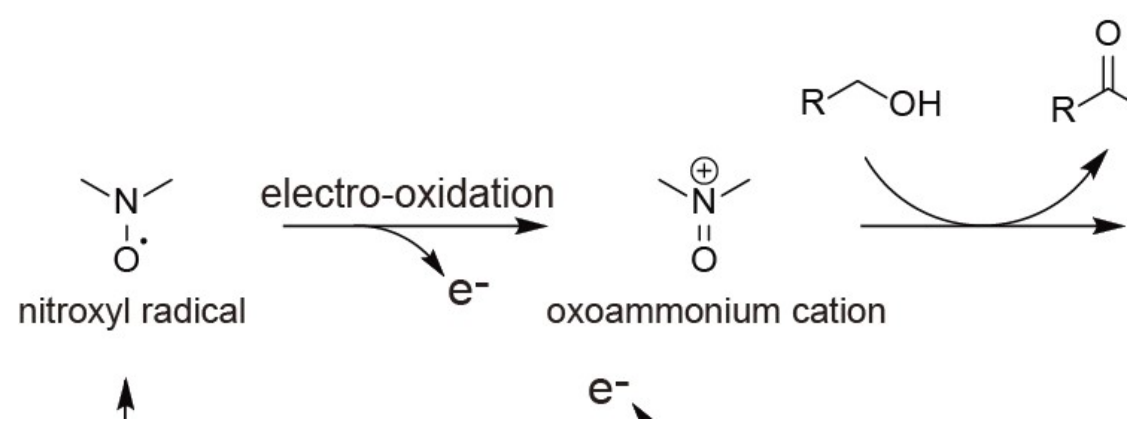

Figure 1. Alcohol detection scheme using nitroxyl radical compounds. 


\section{Materials and Methods}

\subsection{Materials}

TEMPO, A-TEMPO, AZADOL and NHPI were purchased from Tokyo Chemical Industry Co., Ltd. (Tokyo, Japan). AZADOL is reported to be converted to AZADO by oxidation and has comparable oxidation activity for alcohols [18-20]. NNO [11] and Nor-AZADO [21] were synthesized according to the literature. Cyclosporin A was purchased from Nacalai Tesque, Inc. (Kyoto, Japan). The chemical structures of the nitroxyl radical compounds and NHPI used in this study are shown in Figure 2.

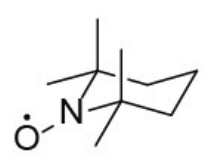

TEMPO

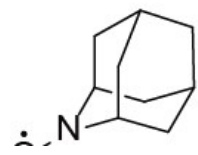

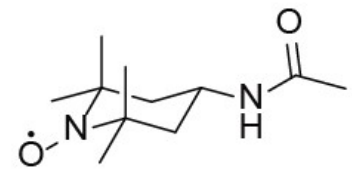

\section{A-TEMPO}

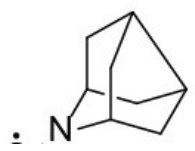

Figure 2. Chemical structures of the nitroxyl radical compounds and NHPI used in this study.

\subsection{Electrochemical Measurements}

Cyclic voltammetry (CV) was performed using an electrochemical analyzer (ALS model 660B, BAS, Tokyo, Japan) in a three-electrode cell comprising a Au electrode (diameter: $3 \mathrm{~mm}$ ) as a working electrode, a platinum wire as a counter electrode, and a $\mathrm{Ag} / \mathrm{Ag}^{+}$reference electrode. Electrochemical measurements were conducted in acetonitrile containing $100 \mathrm{mM}$ tetrabutylammonium perchlorate (TBAP). The third CV cycle was recorded. Measurements were carried out several times and the average values were plotted. All experiments were performed at room temperature (ca. $20{ }^{\circ} \mathrm{C}$ ).

\subsection{Nitroxyl Radical-catalyzed Alcohol Oxidation with an Organic Oxidant}

The catalytic activities of TEMPO, AZADO, and NNO for alcohol oxidation with an organic oxidant were evaluated according to the following reaction procedure. To a solution of an alcohol (3phenyl-1-propanol or 4-phenyl-2-butanol; $1.0 \mathrm{mmol}$ ) and a catalyst (TEMPO, AZADO, or NNO; 10 $\mu \mathrm{mol})$ in dichloromethane $(0.50 \mathrm{~mL})$, was added (diacetoxyiodo)benzene $(1.1 \mathrm{mmol})$ at room temperature (ca. $20^{\circ} \mathrm{C}$ ). The mixture was stirred at the same temperature. Reactions were monitored by gas chromatography (GC) performed on an Agilent 7890A GC system with HP-5 capillary column (0.32 mm × $30 \mathrm{~m}, 0.25 \mu \mathrm{m}$, Agilent Technologies).

\section{Results and Discussion}

\subsection{Evaluation of Alcohol Oxidation of Nitroxyl Radical Compounds}

TEMPO has poor reactivity due to the four adjacent methyl groups required to stabilize the nitroxyl radicals present in the molecule. Iwabuchi developed AZADO as a nitroxyl radical with reduced bulk [20]. However, few examples of the catalytic performance of AZADO under electrochemical conditions have been reported. 
Figure 3 shows cyclic voltammograms for NNO in the presence of various alcohols. The oxidation current of $+0.4 \mathrm{mV}$ vs. $\mathrm{Fc} / \mathrm{Fc}^{+}$increased in the presence of alcohols. The alcohol oxidation capacity was evaluated as $\Delta \mathrm{Ip}$, which is the difference between the oxidation current in the presence and absence of alcohol. Upon applying an electric potential, the nitroxyl radical of NNO is oxidized to become an oxoammonium ion and is thus activated $[22,23]$. The oxoammonium ion is reduced by the alcohol in the solution and then oxidized again by the electrode to regenerate the oxoammonium ion [16]. If this cycle is fast enough compared to the CV sweep rate, $\Delta \mathrm{Ip}$ increases [24, 25].

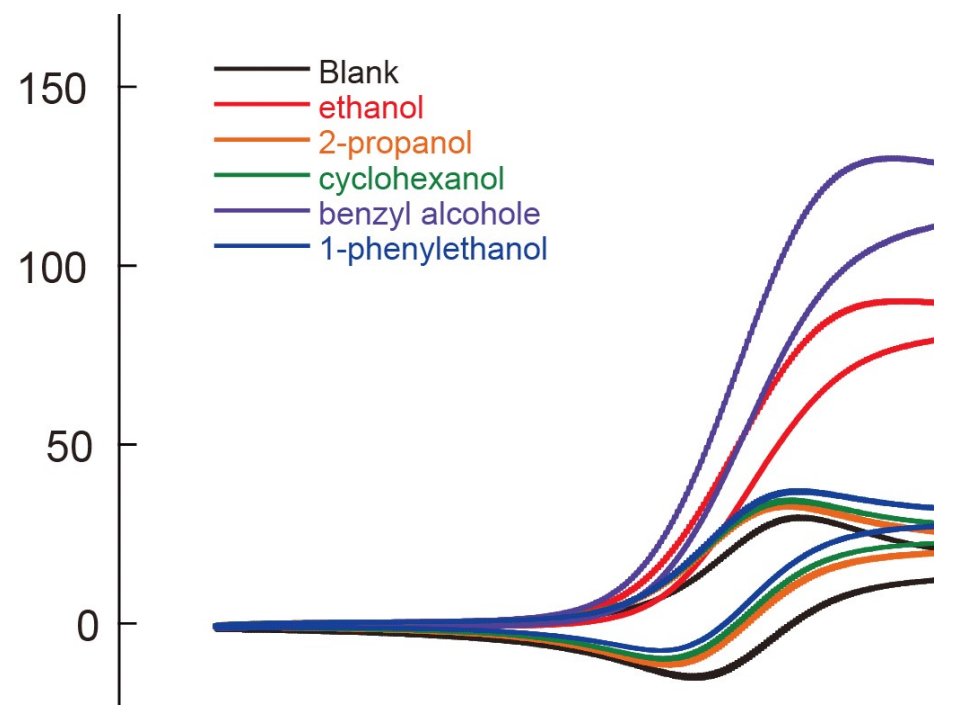

Figure 3. Cyclic voltammograms for NNO $(1.0 \mathrm{mM})$ in the absence and presence of $10 \mathrm{mM}$ ethanol, 2-propanol, cyclohexanol, benzyl alcohol and 1-phenylethanol in acetonitrile (20 mM 2,6-lutidine, $100 \mathrm{mM}$ TBAP). The scan rate was $100 \mathrm{mV} \mathrm{s}^{-1}$.

To evaluate the reactivity of various nitroxyl radical compounds, the $\Delta \mathrm{Ip}$ values for $10 \mathrm{mM}$ of different alcohols in acetonitrile are summarized in Table 1. The alcohols used were ethanol, 2propanol, cyclohexanol, benzyl alcohol, and 1-phenylethanol, with sweep rates of 10, 50 and $100 \mathrm{mV}$ $\mathrm{s}^{-1}$. NNO showed the highest reactivity among the measured nitroxyl radical compounds and the highest current response, not only for primary alcohols such as ethanol and benzyl alcohol, but also for secondary alcohols such as 2-propanol. When the scan rate was increased to $100 \mathrm{mV} \mathrm{s}^{-1}$, no significant change in the current was observed for NNO. In contrast, no change in the oxidation current for TEMPO, which is widely used in existing methods, was observed for any of the added alcohols in the present study. Therefore, A-TEMPO with an acetamide group at position 4 was used to improve the reactivity of the radicals, and the electro-oxidation proceeded. Since TEMPO has a dimethyl group at the 2 position, it is thought that the oxidation reaction does not progress as smoothly as it should near the reaction point. Therefore, similar measurements were performed using AZADO, which has a bicyclic skeleton with reduced TEMPO bulk. It was found that oxidation proceeded with primary alcohols, but not with secondary alcohols. Nor-AZADO with reduced bulk than AZADO also showed an increased current change. However, like AZADO, oxidation did not occur with secondary alcohols and the oxidation current decreased as the sweep rate was increased. NHPI is known to oxidize alcohols by a different reaction pathway than other nitroxyl radical compounds [26,27]. In NHPI, oxidation proceeded with all alcohols except for ethanol.

The measured $\Delta \mathrm{Ip}$ values for various nitroxyl radical compounds were compared using benzyl alcohol at $50 \mathrm{mV} \mathrm{s}^{-1}$. While oxidation did not progress in TEMPO, there was a slight improvement of 
3.4 $\mu \mathrm{A}$ in A-TEMPO, $3.9 \mu \mathrm{A}$ in AZADO, and a fourfold increase to $12.4 \mu \mathrm{A}$ in Nor-AZADO. The NHPI showed a slight improvement to $5.8 \mu \mathrm{A}$. However, NNO showed high reactivity with all alcohols, with a value of $108.8 \mu \mathrm{A}$ for benzyl alcohol, nearly 30 times higher than that for A-TEMPO. The current change was also observed for secondary alcohols albeit with poor reactivity, and $\Delta \mathrm{Ip}$ values of 4.7, 7.3 and 11.0 $\mu \mathrm{A}$ were measured for 2-propanol, cyclohexanol and 1-phenylethanol, respectively.

Table 1. Change in oxidation current $(\triangle \mathrm{I} \mathrm{p} / \mu \mathrm{A})$ for alcohols using various nitroxyl radical compounds (1 $\mathrm{mM}$ nitroxyl radical and $10 \mathrm{mM}$ alcohol in acetonitrile with 2 equivalents of 2,6-lutidine and $100 \mathrm{mM}$ TBAP).

\begin{tabular}{lccccccc}
\hline & Scan rate $\left(\mathrm{mV} \mathrm{s}^{-1}\right)$ & NNO & AZADO & Nor-AZADO & TEMPO & A-TEMPO & NHPI \\
\hline Ethanol & 10 & 64.9 & 1.0 & 7.6 & - & 2.9 & - \\
& 50 & 69.0 & - & 4.7 & - & 3.6 & - \\
& 100 & 62.2 & - & 4.3 & - & 2.4 & - \\
\hline 2-Propanol & 10 & 7.0 & - & - & - & 3.1 & 1.3 \\
& 50 & 4.7 & - & - & - & 3.8 & 1.3 \\
& 100 & 3.8 & - & - & - & 3.4 & 1.3 \\
\hline Cyclohexanol & 10 & 10.2 & - & - & - & 2.9 & 2.0 \\
& 50 & 7.3 & - & - & - & 3.5 & 1.4 \\
& 100 & 6.0 & - & - & - & 3.2 & 1.4 \\
\hline Benzyl alcohol & 10 & 94.0 & 6.0 & 19.8 & - & 3.5 & 9.4 \\
& 50 & 108.8 & 3.9 & 12.4 & - & 3.4 & 5.8 \\
& 100 & 104.8 & 4.4 & 10.2 & - & 3.5 & 5.0 \\
\hline 1-Phenylethanol & 10 & 15.7 & - & - & - & 3.2 & 7.1 \\
& 50 & 11.0 & - & - & - & 2.7 & 4.3 \\
& 100 & 8.8 & - & - & - & 2.1 & 3.7 \\
\hline
\end{tabular}

A hyphen means that an electrocatalytic response was not detected.

AZADO and Nor-AZADO with less-hindered nitroxyl radical moieties exhibited high catalytic activity for alcohol oxidation in organic synthesis [19-21]. Interestingly, under the CV conditions, AZADO showed little anodic current and Nor-AZADO showed a much smaller anodic current than NNO. These results prompted us to compare the catalytic activities of NNO and AZADO for alcohol oxidation with a chemical oxidant. The catalytic activities of NNO, AZADOL, and TEMPO for oxidation of primary and secondary alcohols were compared using (diacetoxyiodo)benzene as the terminal oxidant (Table 2) [28]. For the oxidation of 3-phenyl-1propanol (a primary alcohol), NNO completed the oxidation process within the shortest reaction time (2 hours) (entry 1). AZADO and TEMPO oxidized the same substrate completely within 6 and 9 hours, respectively (entries 2 and 3). On the other hand, NNO was deactivated within 3 hours in the oxidation of 4-phenyl-2-butanol (a secondary alcohol) (entry 4). AZADO completed the oxidation of the same substrate within 4 hours (entry 5). TEMPO showed a slow reaction rate for the oxidation of the secondary alcohol to afford $67 \%$ conversion in 18 hours (entry 6). These results indicate that NNO has higher catalytic activity than AZADO even with a chemical oxidant, but deactivation of NNO could compete in the case of oxidation of a secondary alcohol. The deactivation is most likely due to the oxidation of NNO to a keto-form, which would readily undergo a retro-Michael reaction (Figure 4). 
Table 2. Comparison of catalytic efficiencies of nitroxyl radicals with an organic oxidant.

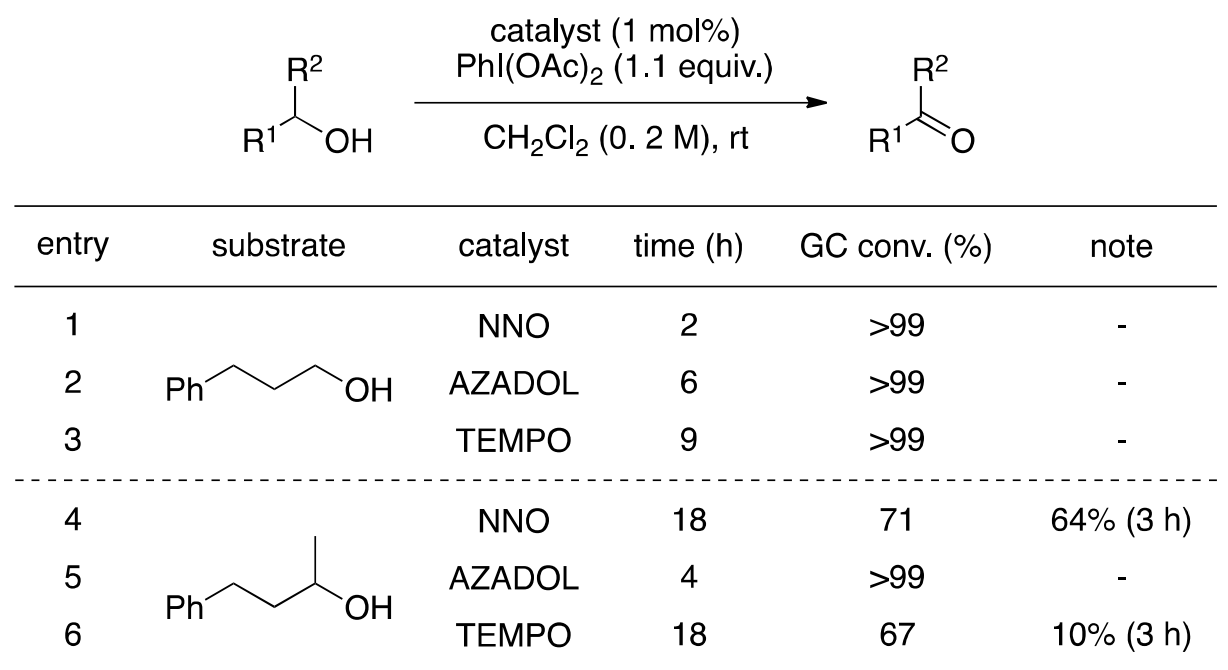

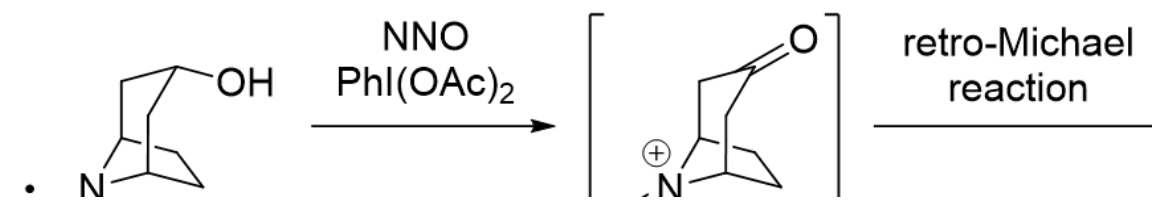

Figure 4. A plausible pathway for deactivation of NNO.

\subsection{Electrochemical Detection of CSA by NNO}

The previous evaluation confirmed that NNO has a high oxidation capacity for alcohols in acetonitrile, and the oxidation current suggested the possibility of quantitative analysis. Cyclosporin $\mathrm{A}$ is a cyclic polypeptide antibiotic produced by fungi and has one secondary hydroxyl group in the molecule. However, due to the complexity of the structure, the reactivity is poor and it is difficult to detect using chemical reactions. Nitroxyl radical compounds oxidize hydroxyl groups, and the detected oxidation current can be used to quantify the compounds. However, the conventional reaction with TEMPO does not allow the oxidation of secondary alcohols to proceed. Therefore, we attempted to measure the electrochemistry of CSA using NNO (Fig. 5). The results showed an increase in oxidation current at CSA concentrations above $1 \mu \mathrm{M}$, with a $\Delta \mathrm{Ip}$ of $0.72 \mu \mathrm{A}$ at $10 \mu \mathrm{M}$ and $1.90 \mu \mathrm{A}$ at $100 \mu \mathrm{M}$. A plot of $\Delta \mathrm{Ip}$ at $+0.4 \mathrm{~V}$ vs. $\mathrm{Fc} / \mathrm{Fc}^{+}$against CSA concentration is shown in Fig. 5 . Since the effective blood concentration of CSA varies depending on the type of disease and on the time of the post-operative course for immunosuppression after organ transplantation, detection in the range of approximately $50-1200 \mathrm{ng} \mathrm{mL}^{-1}(41.6-998 \mu \mathrm{M})$ is required [29-30]. The detection range for the calibration curve obtained in the present study was 1-100 $\mu \mathrm{M}$, which is a sufficient response. 


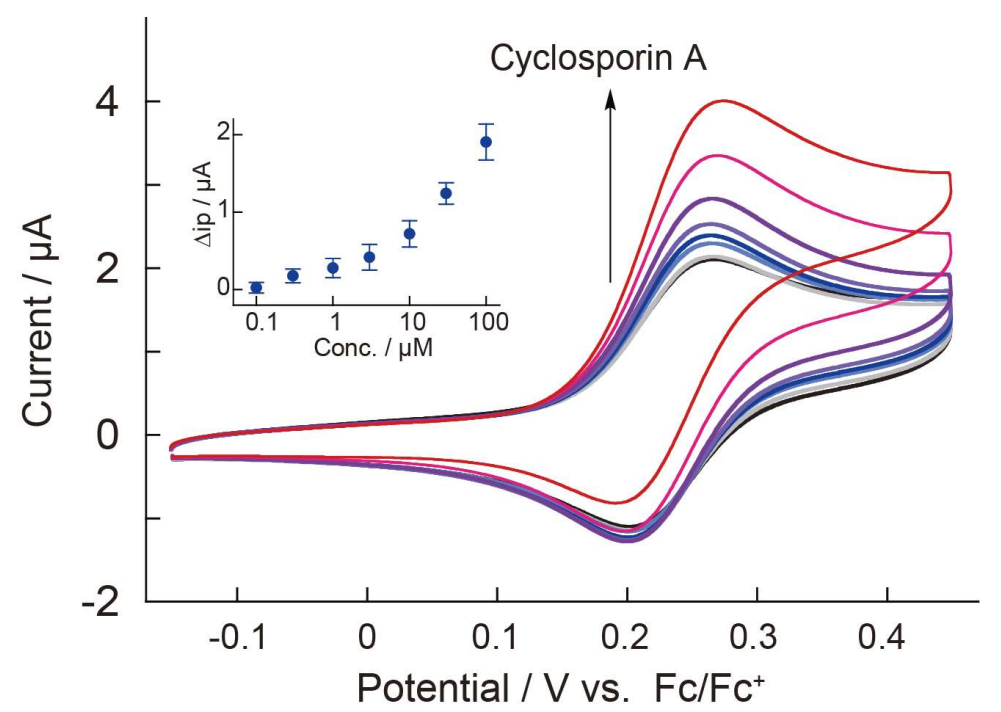

Figure 5. Cyclic voltammograms for $\mathrm{NNO}(0.1 \mathrm{mM})$ in the presence of CSA at a scan rate of $50 \mathrm{mV} \mathrm{s}^{-1}$. The inset shows a calibration curve for CSA created from changes in the oxidation current for NNO.

\section{Conclusions}

The electrochemical oxidation capacity of various nitroxyl radical compounds for alcohols in acetonitrile was evaluated. A high response current was obtained with NNO. The electrochemical measurement of CSA, a TDM drug, was successfully carried out. The detection range was 1-100 $\mu \mathrm{M}$. This performance is sufficient for clinical testing when combined with a rapid pretreatment method.

Author Contributions: Conceptualization and methodology, K.S.; validation and formal analysis, S.K., K.S., K.S., and Y.K.; investigation, S.K, K.S., and M.K.; resources, K.S., K.W., and Y.K.; data curation and writingoriginal draft preparation, K.S.; writing-review and editing, Y.S., K.Y., T.O., Y.I., T.F., K.S., and Y.K.; supervision and project administration, K.S. and Y.K.; funding acquisition, K.S. and Y.K.

Funding: This work was partially supported by a Japan Society for the Promotion of Science Grants-in-Aid for Scientific Research (No. 20K06984) and by the Foundation for Ohu University Joint Research Fund.

Acknowledgments: This work was partially supported by a Japan Society for the Promotion of Science Grantsin-Aid for Scientific Research (No. 20K06984) and by the Foundation for Ohu University Joint Research Fund.

Conflicts of Interest: The authors declare no conflict of interest.

\section{References}

1. Tedesco, D.; Haragsim, L. Cyclosporine: a review. J. Transplant. 2012, 230386.

2. Kagawa, Y.; Yanagawa, M.; Muraki, Y.; Iwamoto, T.; Mizutani, H.; Sugimura, Y.; Kojima, M. Comparison of cyclosporine concentrations in renal transplant recipients using ACMIA and mFPIA methods. Clin. Biochem. 2004, 37, 1016-1021.

3. Lee, Y.W. Comparison between ultra-performance liquid chromatography with tandem mass spectrometry and a chemiluminescence immunoassay in the determination of cyclosporin A and tacrolimus levels in whole blood. Exp. Ther. Med. 2013, 6, 1535-1539.

4. Vogeser, M.; Shipkova, M.; Rigo-Bonnin, R.; Wallemacq, P.; Orth, M.; Widmann, M.; Verstraete, A.G. Multicenter analytical evaluation of the automated electrochemiluminescence immunoassay for cyclosporine. Ther. Drug Monit. 2014, 36, 640-650.

5. Fung, A.W.S.; Knauer, M.J.; Blasutig, I.M.; Colantonio, D.A.; Kulasingam, V. Evaluation of electrochemiluminescence immunoassays for immunosuppressive drugs on the Roche cobas e411 analyzer. F1000Research 2017, 6:1832.

6. Ceglarek, U.; Lembcke, J.; Fiedler, G.M.; Werner, M.; Witzigmann, H.; Hauss, J.P.; Thiery, J. Rapid simultaneous quantification of immunosuppressants in transplant patients by turbulent flow chromatography combined with tandem mass spectrometry. Clin. Chim. Acta 2004, 346, 181-190. 
7. McShane, A.J.; Bunch, D.R.; Wang, S. Therapeutic drug monitoring of immunosuppressants by liquid chromatography-mass spectrometry. Clin. Chim. Acta 2016, 454, 1-5.

8. Polledri, E.; Mercadante, R.; Fusarini, C.F.; Maiavacca, R.; Fustinoni, S. Immunosuppressive drugs in whole blood: validation of a commercially available liquid chromatography/tandem mass spectrometry kit and comparison with immunochemical assays. Rapid Commun. Mass Spectrom. 2017, 31, 1111-1120.

9. Clark, L.D.; Lyons, C. Electrode systems for continuous monitoring in cardiovascular surgery. Ann. N. Y. Acad Sci. 1962, 102, 29-45.

10. Updike, S.J.; Hicks, G.P. The enzyme electrode. Nature, 1967 214, 986-988.

11. Sato, K.; Ono, T.; Yoshida, K.; Ito, T.; Kashiwagi, Y. Electrochemical determination of D-glucose using nortropine- $N$-oxyl under physiological conditions. Electroanaysis 2015, 27, 2272-2274.

12. Golubev, V.A.; Rozantsev, E.G.; Neiman, M.B. Some reactions of free iminoxyl radicals with unpaired electron participation. Izv. Akad. Nauk USSR. Ser. Khim. 1965, 1927-1936.

13. Bobbitt, J.M.; Flores, M.C.L. Organic nitrosonium salts as oxidants in organic chemistry. Heterocycles 1988, 27, 509-533.

14. Cella, J.A.; Kelly, J.A.; Kenehan, E.F. Nitroxide-catalyzed oxidation of alcohols using m-chloroperbenzoic acid. New method. J. Org. Chem. 1975, 40, 1860-1862.

15. Semmelhack, M.F.; Chou, C.S.; Cortes, D.A. Nitroxyl-mediated electrooxidation of alcohols to aldehydes and ketones. J. Am. Chem. Soc. 1983, 105, 4492-4494.

16. Semmelhack, M.F.; Schmid, C.R.; Cortes, D.A. Mechanism of the oxidation of alcohols by 2,2,6,6tetramethylpiperidine nitrosonium cation. Tetrahedron lett. 1986, 27, 1119-1122.

17. Yamauchi, Y.; Maeda, H.; Ohmori, H. Amperometric detection of alcohols and carbohydrates coupled with their electrocatalytic oxidation by 2,2,6,6-tetramethylpiperidinyl-1-oxy (TEMPO). Chem. Pharm. Bull. 1996, 44, 1021-1025.

18. Sasano, Y.; Sato, H.; Tadokoro, S.; Kozawa, M.; Iwabuchi, Y. Safe and scalable aerobic oxidation by 2azaadamantan-2-ol (AZADOL)/NOx catalysis: Large-scale preparation of Shi's catalyst. Org. Process Res. Dev. 2019, 23, 571-577.

19. Iwabuchi, Y. Discovery and exploitation of AZADO: the highly active catalyst for alcohol oxidation. Chem. Pharm. Bull. 2013, 61, 1197-1213.

20. Shibuya, M.; Tomizawa, M.; Suzuki, I.; Iwabuchi, Y. 2-Azaadamantane N-Oxyl (AZADO) and 1-MeAZADO: Highly efficient organocatalysts for oxidation of alcohols. J. Am. Chem. Soc 2006, 128, 8412-8413.

21. Hayashi, M.; Sasano, Y.; Nagasawa, S.; Shibuya, M.; Iwabuchi, Y. 9-Azanoradamantane N-oxyl (NorAZADO): a highly active organocatalyst for alcohol oxidation. Chem. Pharm. Bull. 2011, 59, 1570-1573.

22. Summermann, W.; Deffner, U. Electrochemical oxidation of aliphatic nitroxyl radicals. Tetrahedron 1975, 31, 593-596.

23. Kaifer, A.E.; Bard, A.J. Polymer films on electrodes. 20. An ESR study of several spin probes incorporated into Nafion. J. Phys. Chem. 1986, 90, 868-873.

24. Murray, R.W. Chemically modified electrodes, in: Electroanalytical Chemistry, vol. 13, Marcel Dekker, New York, 1984, p. 191-368.

25. Murray, R.W. Molecular design of electrode surface, in: Techniques of Chemistry, vol. 22, Wiley-Interscience, New York, 1992, p. 1-48.

26. Ishii, Y.; Sakaguchi, S.; Iwahama, T. Innovation of hydrocarbon oxidation with molecular oxygen and related reactions. Adv. Synth. Catal. 2001, 343, 393-427.

27. Recupero, F.; Punta, C. Free radical functionalization of organic compounds catalyzed by $N$ hydroxyphthalimide. Chem. Rev. 2007, 107, 3800-3842.

28. De Mico, A.; Margarita, R.; Parlanti, L.; Vescovi, A.; Piancatelli, G. A versatile and highly selective hypervalent iodine(III)/2,2,6,6-tetramethyl-1-piperidinyloxyl-mediated oxidation of alcohols to carbonyl compounds. J. Org. Chem. 1997, 62, 6974-6977.

29. Barakat, O.; Peaston, R.; Rai, R.; Talbot, D.; Manas, D. Clinical benefit of monitoring cyclosporine C2 and $\mathrm{C} 4$ in long-term liver transplant recipients. Transplant Proceedings 2002, 34, 1535-1537.

30. Perner, F. Am J Transplant 2002, 2, 148-156.

31. Author 1, A.; Author 2, B. Title of the chapter. In Book Title, 2nd ed.; Editor 1, A., Editor 2, B., Eds.; Publisher: Publisher Location, Country, 2007; Volume 3, pp. 154-196. 